\title{
828 QUANTIFYING PERIVASCULAR IMMUNE CELLS IN THE STROMA OF HUMAN TRIPLE NEGATIVE BREAST TUMORS USING DEEP LEARNING SPATIAL ANALYTICS
}

${ }^{1}$ Anna Juncker-Jensen, ${ }^{1}$ Nicholas Stavrou*, ${ }^{2}$ Mohammed Moamin, ${ }^{1}$ Mate Nagy,

${ }^{2}$ Richard Allen, ${ }^{2}$ Angela Cox, ${ }^{2}$ Claire Lewis. ${ }^{1}$ NeoGenomics, Aliso Viejo, CA, United States;

${ }^{2}$ University of Sheffield, Sheffield, United Kingdom

Background The spatial organization and density of the immune infiltrate in the tumor microenvironment, referred to as immune contexture, can yield information relevant to prognosis and prediction of response to immunotherapy in cancer. Specifically, a distinct subset of tumor-associated macrophages (TAMs) accumulate around blood vessels where they stimulate tumor angiogenesis and limit tumor responses to frontline anti-cancer therapies like irradiation and chemotherapy.

Methods In this study we leveraged the NeoGenomics MultiOmyx Multiplex Immunofluorescence platform alongside artificial intelligence (AI) based quantitative image analysis. This AI platform was ultimately used to investigate the distribution of perivascular (PV) TAMs, CD4+ and CD8 + T cells, and CD4 +FOXP3 + regulatory $\mathrm{T}$ cells (Tregs) of 40 human triple negative breast carcinomas (TNBCs), and how this changed following neoadjuvant chemotherapy. During the multiplexing phase, eleven rounds of paired antibody staining were performed in sequence on tumor sections. After each round of staining, high resolution images were captured for regions of interests (ROIs) selected by a pathologist. We used AI models to segment and classify cells for each biomarker and classify regions as tumor cell islands (TCIs) or stroma. First, each nucleus was segmented out using a convolutional neural network combined with watershed thresholding on the DAPI (diamidino-2-phenylindole) immunofluorescent image. From the resulting nuclear segmentation mask, a pixel dilation on cells classified as nontumor was employed to generate a cellular segmentation mask. A list of neighbours within a specified distance for each cell was generated by radially expanding from the cellular segmentation mask. Finally, cell neighbour information was combined with the marker expression information to quantify the cell clusters of interest.

Results We discovered that in the PV areas, up to $30 \%$ of PD1-LAG3-CD3+CD8 $+\mathrm{T}$ cells formed direct contact with both CD163+TIM3 + TAMs and CD4+FOXP3 + Tregs. Furthermore, these immune cell triads preferentially accumulated in the PV stroma regions. It is likely that close interaction with immunosuppressive TAMs and Tregs would supress the function of $\mathrm{T}$ cells as they enter the PV region to reach the TCIs.

Conclusions Using an advanced analytics platform, we invented a new method to quantify clusters of cells within various regions of a tumor section. Using this platform, we detected specific immune cell triads, the frequency and location of which could correlate with the efficacy of T-cell based immunotherapies in TNBC. These analyses will enable further investigation of numerous complex cell interactions in TMEs.

http://dx.doi.org/10.1136/jitc-2021-SITC2021.828 